\title{
Piceatannol-mediated alteration of amyloidogenesis in SH-SY5Y neuroblastoma cells treated with amyloid-beta
}

\author{
Yun Jung Yang, Hee Yun Cha and Soo Jin Yang \\ Seoul Women's University, Seoul, Korea, Republic of
}

\begin{abstract}
High glucose condition impairs neuronal integrity and function by regulating amyloidogenesis and neuroinflammation. Here, we pursue to investigate whether high glucose condition leads impairments in neuronal integrity/function by inducing amyloidogenesis and neuroinflammation, and whether piceatannol (PIC) restores high glucose condition-induced deteriorations in neuronal cells. High glucose condition was induced by maintaining SH-SY5Y neuroblastoma cells in $25 \mathrm{mM}$ glucose. In addition, amyloid-beta was applied to make the cells having amyloidogenesis-mediated deleterious alterations. Cells were maintained in low glucose (2.5 mM glucose) or high glucose $(25 \mathrm{mM}$ glucose) condition. And, two doses $(10$ and $20 \mu \mathrm{M})$ of PIC and/or $10 \mu \mathrm{M}$ of amyloid-beta were treated in a subset of cells for $24 \mathrm{~h}$. There were no significant differences in cell morphology and cell viability among groups. PIC treatment did not show cell toxicity based on cell viability assessed by PrestoBlue assay. High concentration of glucose and amyloid-beta treatments increased amyloid-beta concentrations in cell lysates and conditioned media, which were reduced by PIC. $10 \mu \mathrm{M}$ PIC treatment for $24 \mathrm{~h}$ decreased amyloid precursor protein (APP), beta-site amyloid precursor protein cleaving enzyme-1, presenilin (PS) 1 and tau. Among analyzed inflammatory markers, interleukin (IL)-1 beta, IL-6, and tumor necrosis factor (TNF)-alpha were reduced in conditioned media by $10 \mu \mathrm{M}$ PIC administration for $24 \mathrm{~h}$. These data indicate that high glucose condition may result in excessive levels of amyloidogenesis and neuroinflammation as well as subsequent changes in neuronal integrity/function, and that PIC treatment may ameliorate the deleterious consequences from high glucose and amyloid-beta treatments.
\end{abstract}

\section{Conflict of Interest}

There is no conflict of interest. 